\title{
Micro-porosity as damage indicator for characterizing cyclic thermal shock-induced anisotropic damage in oxide/oxide ceramic matrix composites
}

\author{
Zhengmao Yang ${ }^{\mathrm{a}}$, Hui Liu ${ }^{\mathrm{b}}$, Huang Yuan ${ }^{\mathrm{b}, *}$ \\ ${ }^{a}$ Institute of Mechanics, China Academy of Sciences, Beijing, China \\ ${ }^{\mathrm{b}}$ School of Aerospace Engineering, Tsinghua University, Beijing, China
}

\section{A R T I C L E I N F O}

\section{Keywords:}

Ceramic matrix composite

Cyclic thermal shock

Micromechanical damage

Thermo-mechanical damage

Hierarchical porosity

\begin{abstract}
A B S T R A C T
Ceramic matrix composites display complex mechanical behavior under thermo-mechanical loading conditions. The present work focuses on micro-structural evolution and resultant macroscopic property representation of the composites after cyclic thermal shocks. Micro-structural investigation reveals that variations of the hierarchical porosity in the matrix characterize the collective behavior of micro-structural evolution in the material. Cyclic thermal shocks induce thermo-mechanical damage and lead to increasing matrix porosity. Material damage development is driven by elastic strain energy density which is related to the matrix porosity increment. Experiments confirm that the anisotropic damage can be represented by the porosity increment accurately. The porosity provides a meaningful model for the thermal shock damage evolution in the anisotropic composites.
\end{abstract}

\section{Introduction}

The ceramic matrix composite (CMC) is a light-weight structural material that possesses high specific strength at high temperature up to $1200^{\circ} \mathrm{C}$, which makes it interesting for hot-section components in gas turbine engines and thermal protection systems in spaceaccess vehicles [1-3]. For such applications the material has to be additionally suitable for harsh operating conditions besides the high temperature, for instance, it must be thermal shock resistant, oxidation resistant and micro-structural stable [4-6]. The additional obstacle for application of CMC is the limited knowledge in micro-structure evolution and mechanical property degeneration as well as lack of solid mechanics models to predict residual mechanical performance and fatigue life. More essentially, high temperature gradients under severe loading conditions can accelerate the premature failure of CMC due to excessive thermal stresses and accompanying microstructural changes [7-9]. To establish a reliable fatigue life assessment method for CMC, more comprehensive knowledge of the mechanism of the thermo-mechanical damage is necessary [10], and even more important are the thermal shockinduced thermo-mechanical damage evolution and its effects on the mechanical behavior of CMC.

Cyclic thermal shocks-induced thermo-mechanical damage mechanisms and damage accumulations in continuous fiber-reinforced CMC have been the subject in numerous investigations [11,12]. However, published works concentrated only on studying damage mechanisms, and did not develop correlations among microstructure, mechanical behavior and thermo-mechanical mechanisms. Recently Yang et al. [13-15] studied cyclic thermal shock-induced thermo-mechanical damage in CMC and found that the macroscopic damage is mainly caused by micro-cracks in the matrix induced by thermal stresses during shocks. The material

\footnotetext{
* Corresponding author.

E-mail address: yuan.huang@tsinghua.edu.cn (H. Yuan).
} 


\section{Nomenclature}

A cross-sectional area in the tensile direction of the specimen

$A_{\text {crack }} \quad$ total crack surface in the loading direction

$B_{\mathrm{i}} \quad$ Biot modulus

$D$ damage variable

$E_{0} \quad$ initial elastic modulus

E the residual elastic modulus

$E_{\mathrm{Up}}$ the theoretical upper bound of the composite elastic modulus obtained from the rule of mixture

$E_{\text {Low }}$ the theoretical lower bound of the composite elastic modulus obtained from the rule of mixture $\bar{E}_{\mathrm{f}} \quad$ the nominal elastic modulus of the fiber phase

$\bar{E}_{\mathrm{m}} \quad$ the nominal elastic modulus of the matrix phase

$G \quad$ energy release rate

$G_{12} \quad$ in-plane shear modulus

$h \quad$ heat transfer coefficient

$k$ thermal conductivity

$l \quad$ the half thickness of the thin plate specimen

$m$ model parameter

$n_{\mathrm{f}} \quad$ model parameter

$n_{\mathrm{m}} \quad$ model parameter

$N \quad$ number of thermal shock cycles

$s$

crack thickness

\section{$T_{\text {shock }}$}

$T_{\text {init }}$

$U$

$V_{\mathrm{f}}$

$V_{\mathrm{m}}$

$\alpha \quad$ model parameter, a dimensionless increasing

thermal shock temperature

thermal shock temperature at which initial thermal shock induced damage occurs

strain energy

the volume fractions of fiber in the composite

the volume fractions of matrix in the composite, and $V_{\mathrm{m}}=1-V_{\mathrm{f}}$ function of the thermal shock temperature, $\ln \left(T_{\text {shock }} / T_{\text {init }}\right)$

$\alpha_{\mathrm{TEC}} \quad$ thermal expansion coefficient (TEC)

$\gamma \quad$ model parameter

$\zeta \quad$ current porosity

$\zeta_{0} \quad$ initial porosity

$\Delta \zeta \quad$ porosity increment

$\zeta_{\mathrm{f}} \quad$ fiber porosity

$\zeta_{\mathrm{m}} \quad$ matrix porosity

$\eta_{0}$ the effects coefficient of the fiber orientation

$\nu$ the Poisson's ratio

$\rho \quad$ the material density

$\varpi$ the weight factor for representing the influence of between fiber phase and matrix phase

$\sigma_{\mathrm{th}} \quad$ the thermal stress

$\sigma_{x} \quad$ the tensile stress

degradation is characterized by reduction of elastic modulus and driven by the elastic strain energy. A damage model was introduced based on experiments within the framework of continuum damage mechanics.

The cyclic thermal shock-induced thermo-mechanical damage mechanism of CMC is micro-cracks nucleation and growth in the matrix as well as fiber-matrix delaminations $[11,13]$. The damage including micro-cracks and interface debonding in composites can be described within the framework of continuum damage mechanics (CDM) when the specimen dimension is sufficiently larger than the cracks $[16,17]$. Matrix cracking can be typically represented by the decreasing stiffness of the material, as reported in [13]. The damage variable of the material degradation can, furthermore, be determined by, e.g. crack density [18-20]. However, determining the damage evolution law usually needs extensive tests on local stresses in the vicinity of the matrix cracks, which is difficult for composite phasess in micron or even impossible. Finding a meaningful damage variable is interesting for developing a quantitative description for the composite degradation.

The hierarchical porosity exists in the matrix of the oxide/oxide ceramic composite materials [13]. It is well documented that the porosity change under thermo-mechanical loading is one of the major features influencing the mechanical performance of composites [21-24]. The porosity plays a dominant role in characterizing mechanical behavior of the material and can be considered as a macroscopic manifestation of cumulative changes in the microstructure of composites.

The objective of the present work is to develop a novel characterization approach of thermo-mechanical damage of CMC composites. Correlation between the micro-structural evolution and the macroscopic mechanical properties was established based on detailed experimental observations. The micro-porosity increment in the matrix is proposed to represent the thermo-mechanical damage and incorporated into the continuum mechanics model of the oxide/oxide ceramic matrix composite.
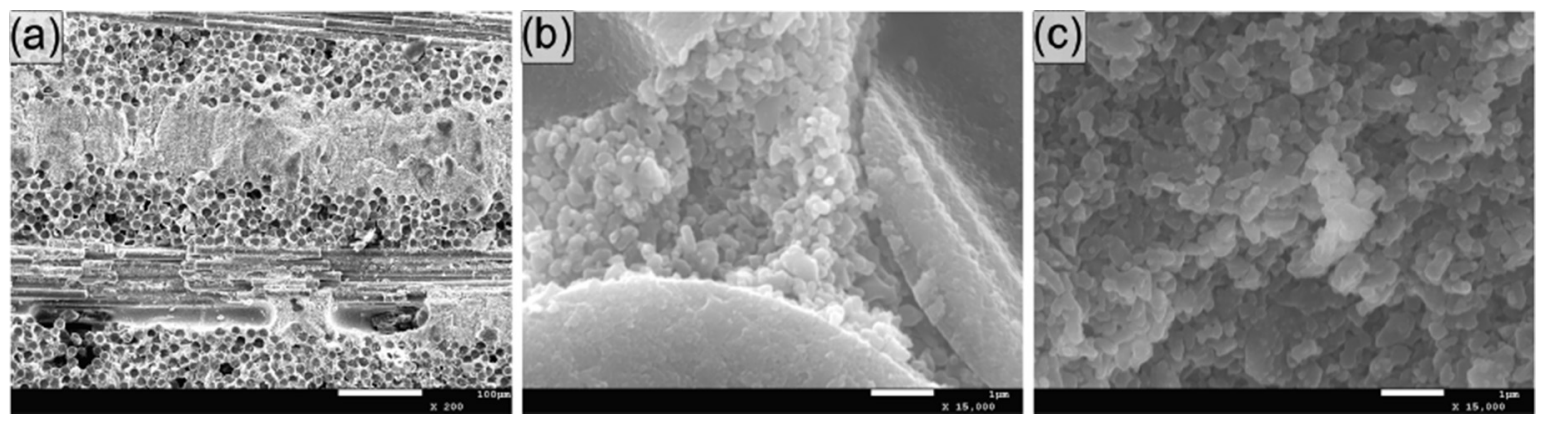

Fig. 1. The in-situ 2-D woven oxide/oxide CMC: (a) Overview; (b) Fiber and matrix; (c) The porous matrix. 


\section{Materials and experiments}

\subsection{Material characterization}

In the present work, the tested material 2-D woven oxide/oxide ceramic matrix composite consists of woven Nextel ${ }^{\mathrm{TM}} 610$ fibers $\left(99 \% \alpha-\mathrm{Al}_{2} \mathrm{O}_{3}, 0.7 \% \mathrm{Fe}_{2} \mathrm{O}_{3}, 0.3 \% \mathrm{SiO}_{2}\right) \mathrm{Al}_{2} \mathrm{O}_{3}-\mathrm{SiO}_{2}-\mathrm{ZrO}_{2}$ matrix with a density of $4.2 \mathrm{~kg} / \mathrm{dm}^{3}$. The as-received material was in $2.8 \mathrm{~mm}$ thick plates. The uncoated Nextel ${ }^{\mathrm{TM}} 610$ fibers with the density of $4 \mathrm{~kg} / \mathrm{dm}^{3}$ are in $0^{\circ} / 90^{\circ}$ woven layers. The fiber volume fraction of CMC is approximately $44 \%$. The matrix has chemical compositions of $85 \% \mathrm{Al}_{2} \mathrm{O}_{3}$ and $15 \% 3 \mathrm{YSZ}$ (in weight). The matrix is porous, as shown in Fig. 1. In Table 1 the thermal expansion coefficient (TEC), elastic modulus as well as the ultimate stress of the chemical product are summarized. Since the matrix contains a high porosity, over $60 \%$, its effective property must be much lower than those in Table 1.

The composite material possesses a density of $2.71 \mathrm{~kg} / \mathrm{dm}^{3}$, so that the total porosity of the material reaches $34 \%$. The mesoscopic picture of the cutting surface in Fig. 1 reveals that there are a lot of cylindrical pores in size of $>100 \mu \mathrm{m}$ diameter oriented in the fiber direction and distributed in the thickness. The smooth uniform microstructure of the pore surface reveals that the pore exists before cutting. All the pores reduce the material stiffness. Two categories of micro-pores were identified: Nano-voids in the matrix and micro-pores existing in the intraply as well as interply. From this observation one may see that CMC contains microscopic pores distributed overall, which can be understood as initial defects.

From Fig. 1 the composite contains different micro-pores. The pores in the matrix, however, are much smaller than the matrix particles and in size less than $50 \mathrm{~nm}$. The matrix is effectively a collection of the alumina particles with a porosity larger than $48 \%$ in the matrix if the particles can be approximated as ellipsoids, which corresponds to a total porosity of $28 \%$ for the nano-voids. This result predicts ca. $7 \%$ micro-pores in fiber plies [14].

In the present work, two types of specimens were investigated in the cyclic thermal shock test. The tensile specimen is machined into the coupon shape with a length of $160 \mathrm{~mm}$ and width of $20 \mathrm{~mm}$ using water jet, as shown in Fig. 2, to identify the stress-strain relationship of the material as well as degradation of the composite property after thermal shocks. The other specimens for the thermal shock study were cut from as-received CMC plates, the dimensions were $20 \mathrm{~mm} \times 15 \mathrm{~mm} \times 2.8 \mathrm{~mm}$ was used to examine the micro-structural evolution in the composite after cyclic thermal shocks and to study the cyclic thermal shock-induced thermomechanical damage mechanism.

\subsection{Cyclic thermal shock tests}

The cyclic thermal shock tests (thermal down shock) to the CMC specimens were performed by heating the specimen up to a given temperature, then isothermal exposure to the high temperature for $10 \mathrm{~min}$ for temperature homogenization and quenched in distilled water. It should be noted that the quench water needs to be continuously stirred to avoid build-up of a gas film on the specimen surface, because bubbles and the vapor layer can change the heat transfer coefficient between the specimen and the quenching medium, which can change the heat transfer mechanism as the shock transient temperature changes. After the specimens had cooled to the room temperature, they were dried, inspected for visible damage (such as macroscopic delamination). The maximum shock temperature is set to $1100^{\circ} \mathrm{C}$ in accordance with the potential application of the material. The thermal process was repeated to study effects of the shock cycles. More details about the thermal shock tests are referred to [13].

Different techniques were used to explore correlations among microstructure, mechanical behavior and damage mechanisms: Scanning electron microscope (SEM), X-ray computed nano-tomography (nano-CT), porosimetry and MTS material testing. Fig. 3 illustrates a typical nano-CT slice of a thermal shocked CMC specimen. To avoid the possible edge effects, a hexahedron region of interest is extracted from the center of the thermal shocked CMC specimen. In the figure, the black phases correspond to matrix cracks and micro-pores. Whereas micro-cracks are from thermal shocks, the micro-pores are formed in material manufacturing and are inherent. The brightest phases are matrix-rich region. The X-ray nano-CT images provide an insight view of the microstructure into the material.

Besides micro-pores mentioned previously, two kinds of failure mechanisms can be found in the 2-D woven oxide/oxide CMC, which affect the material overall property differently: (i) Delamination between the reinforced fibers and matrix within the boundary layer region are caused by high temperature gradients as well as different thermal expansions. (ii) Micro-cracks in the matrix induced by the in-plane tensile stress are distributed along the thickness when the specimen is subjected to thermal shocks. These micro-cracks formed due to the low-toughness and high strength characteristics of the matrix. The matrix crack density increases with thermal shock cycles [14].

Table 1

Mechanical and thermal properties of the oxide/oxide CMC at room temperature $[7,25]$

\begin{tabular}{lccc}
\hline & TEC $\left(10^{-6} /{ }^{\circ} \mathrm{C}\right)$ & $E(\mathrm{MPa})$ & $\sigma_{\mathrm{u}}(\mathrm{GPa})$ \\
\hline $99 \% \alpha-\mathrm{Al}_{2} \mathrm{O}_{3}$ fiber & 8.8 & 373 & 2930 \\
$85 \% \mathrm{Al}_{2} \mathrm{O}_{3}-15 \%$ YYSZ matrix & 10.2 & 210 & $\approx 45$ \\
\hline
\end{tabular}




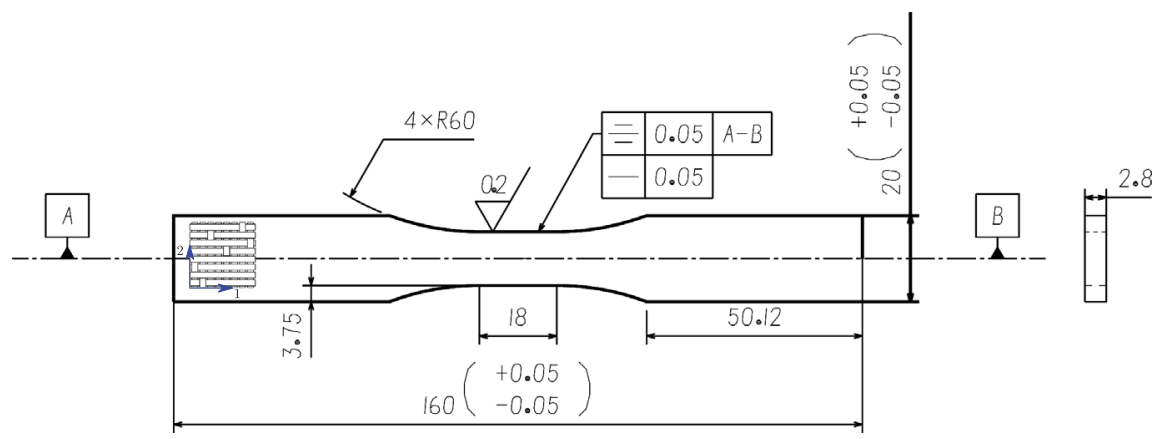

Fig. 2. Uniaxial tension specimen geometry of the oxide/oxide CMC. The warp bundles and the weft bundles represent Direction $1\left(0^{\circ}\right)$ and Direction $2\left(90^{\circ}\right)$, respectively. The weft bundles (direction 1) are nearly straight, whereas the warp bundles (Direction 2) are wavy.
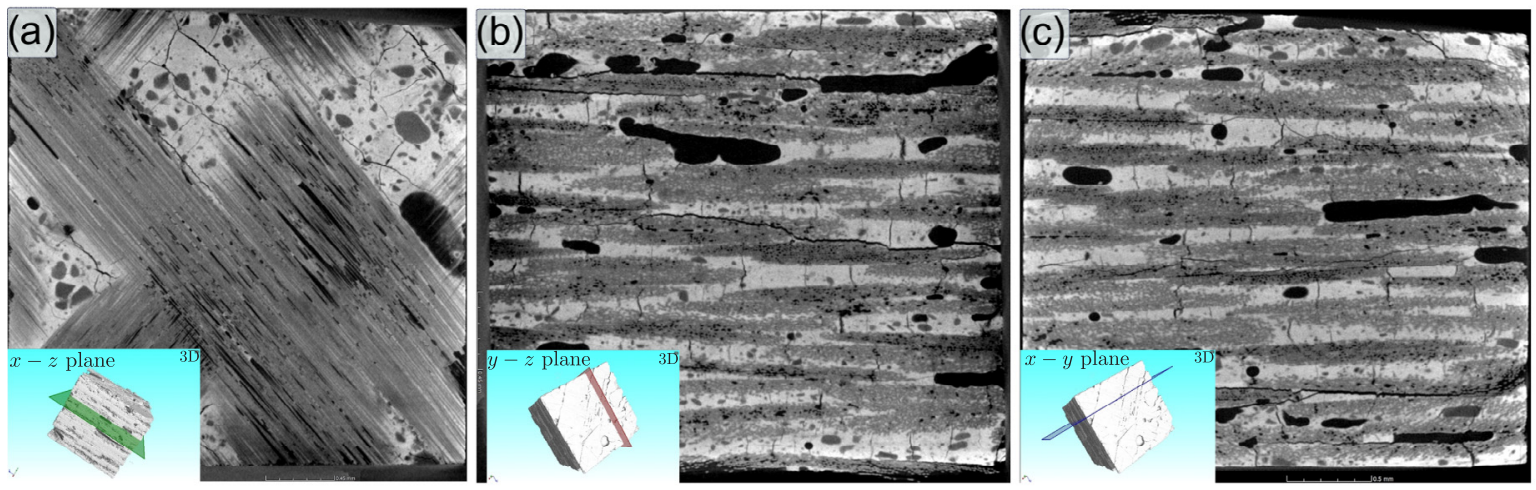

Fig. 3. High resolution X-ray computer tomography of the 2-D woven oxide/oxide CMC specimen after four thermal shocks. Hierarchical porosity exists in the material: The mesoscopic pores were formed during material manufacture process and are typical for the oxide/oxide CMC; the delamination cracks among fiber plies were induced by thermal shocks; the micro pores existed in the matrix and caused by thermo-mechanical loads.

\section{Representations of the thermal shock damage}

\subsection{Characterization of residual mechanical property}

To determine the mechanical property of the composite after cyclic thermal shocks, the specimen was tested in the MTS servohydraulic test machine at room temperature. In the present work, thermo-mechanical damage evolution in the $0^{\circ}$, $45^{\circ}$ and $90^{\circ}$ directions of the 2-D woven oxide/oxide CMC is investigated. Obviously, the tensile behavior in the $0^{\circ}$ and $90^{\circ}$ is dominated by fibers and the material behaves brittle, in which the elastic moduli of the principal directions can be determined. The off-axis tensile behavior in $\pm 45^{\circ}$ is significantly elastic-plastic [15], which provides the shear modulus.

Under an orthotropic plane stress state, the elastic stress-strain relationship can be written as

$$
\left\{\begin{array}{c}
\varepsilon_{1} \\
\varepsilon_{2} \\
\gamma_{12}
\end{array}\right\}=\left[\begin{array}{ccc}
\frac{1}{E_{1}} & -\frac{\nu}{E_{1}} & 0 \\
-\frac{\nu}{E_{1}} & \frac{1}{E_{2}} & 0 \\
0 & 0 & \frac{1}{G_{12}}
\end{array}\right]\left\{\begin{array}{c}
\sigma_{1} \\
\sigma_{2} \\
\sigma_{12}
\end{array}\right\} .
$$

Above $E_{1}$ and $E_{2}$ represent elastic moduli in the principal directions, i.e. the longitudinal 1- and the lateral 2-direction of the material, respectively. $G_{12}$ stands for the in-plane shear modulus, $v$ is Poisson's ratio measured during the tension experiment in the 1 direction.

In the present work, the shear modulus of the material was evaluated from a $45^{\circ}$ off-axis tensile test, in accordance with the ASTM standard D3518 [26]. The axial strain $\varepsilon_{x}$ and the transverse strain $\varepsilon_{y}$ were measured by a strain gage rosette. According to [26], the shear modulus $G_{12}$ can be obtained from

$$
G_{12}=\frac{\sigma_{x}}{2\left(\varepsilon_{x}-\varepsilon_{y}\right)}
$$

where $\sigma_{x}$ notes the tensile stress, defined as $\sigma_{x}=F / A$, where $A$ is the cross-sectional area in the tensile direction of the specimen. The initial elastic moduli are $E_{1}=120.5 \mathrm{GPa}, E_{2}=90 \mathrm{GPa}$ and $G_{12}=46 \mathrm{GPa}$, respectively. In comparing with the elastic moduli of the fiber and matrix, the composite possesses a very low stiffness, which is related to the inhomogeneous and porous microstructure. 
The material degradation from thermal shocks reduces the elastic modulus of the material as well as shear modulus, as shown in Fig. 4(a). In the figure elastic modulus after one cycle of thermal shock is expressed as a function of the thermal shock temperature, $T_{\text {shock}}$. The effect of $T_{\text {shock }}$ on CMC mechanical properties in $0^{\circ} / 90^{\circ}$ fiber orientation is inhomogeneous. The drop in shear modulus reaches $18.5 \%$, whereas $E_{1}$ and $E_{2}$ just reduce $14 \%$ and $8 \%$, respectively. The higher shock temperature results in larger degradation of elastic property. It is interesting that drops of all three modulus occur at the shock temperature of $400{ }^{\circ} \mathrm{C}$.

Thermal shock-induced micro-cracking in matrix results in the composite stiffness decreases [14]. The experiments show, furthermore, as $T_{\text {shock }}$ exceeds $1000^{\circ} \mathrm{C}$, the elastic moduli reach a saturation level, which implies that the material can no longer further damaged.

The situation in cyclic thermal shocks differs from the single shock. Fig. 4(b) illustrates the development of the elastic moduli of the oxide/oxide CMC as functions of the cumulative number of thermal shock cycles, $N$, with $1100^{\circ} \mathrm{C}$. As shown in Fig. $4(\mathrm{~b})$, the overall reduction of $E_{1}$ modulus is higher compared to that of $E_{2}$, and shear modulus seems even more sensitive. After eight shocks the reductions reach a stable stage, near $35 \%$ of the initial moduli.

\subsection{Micro-structural evolution during cyclic thermal shocks}

In the evolution and characterization of residual mechanical properties of the oxide/oxide CMC, it demonstrated the importance of taking micromechanical effects into account to model macroscopic failure of the material. The mercury intrusion porosimetry can measure different porosities as well as generate a pore size distribution of the open porosity [27], by using a Micromeritics Autopore IV 9510 porosimeter. The hierarchical porosity in the CMC matrix can be divided into two different classes:

(a) Nano-pores are considered as pores smaller than $200 \mathrm{~nm}$ in diameter; The nano-pores exist in the matrix between the aluminazirconia particles, with a size up to $200 \mathrm{~nm}$, and the micro-pores are present as manufacturing micro defects. Since the reinforced fibers possess much high strength, the matrix damage is the failure mechanism.

(b) Micro-pores as pores larger than $200 \mathrm{~nm}$ as regards to mercury intrusion. Cracks nucleate in the matrix and propagate between the matrix particles and when cracks reach reinforced fibers, they are deflected at the fibre/matrix interface, which leads to fibre/ matrix debonding and the delamination occurs $[28,29]$.

Macroscopic damage is the collective representation of nucleation and propagation of micro-cracks and macro-cracks in the matrix of CMC. The weak matrix concept assumes the strong fibre/matrix bond, but the high porosity of the matrix, even at the fibre/ matrix interface, ensures a weak fibre/matrix interface.

The nano-voids are inherent and hardly affected the material damage. From micro-scopic observation, it is clear that the thermomechanical damage from cyclic thermal shocks is induced in the form of micro-cracks and delamination. The microscopic evolution of the matrix after thermal shock can be characterized by the increasing porosity due to micro-cracks. Especially, the delamination induces rather large pores into the material.

Fig. 5(a) illustrates the distribution of pores for four different CMC states: The initial state, after one shock, after four shocks and after eight shocks. All measurements confirm a large peak in the pore distribution at ca. $100 \mathrm{~nm}$, which corresponds pores between matrix particles. Interesting is the peak for the eight shocks is shifted slightly, which means the small size increment in the nanovoids. In all specimens the nano-porosity takes the major part in the total porosity, as shown in the accumulative porosity distribution in Fig. 5(b).

Fig. 5(b) reveals that the porosity of the material increases with thermal shock cycles, which implies irreversible micro-structural

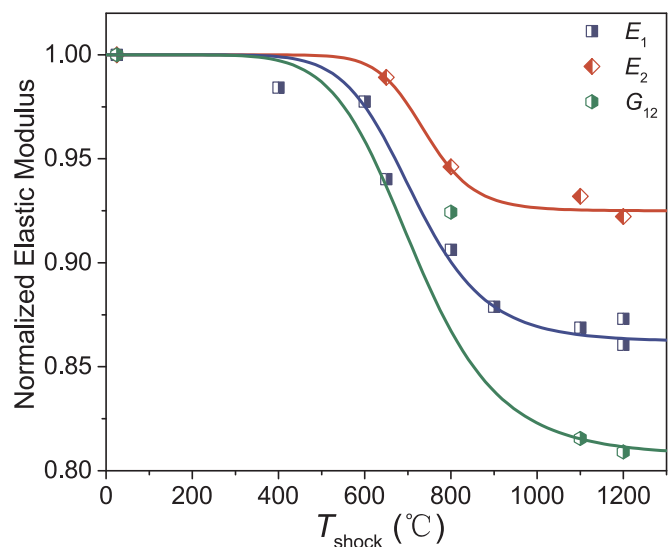

(a) Residual elastic moduli as a function of thermal shock temperature $T_{\text {shock }}$.

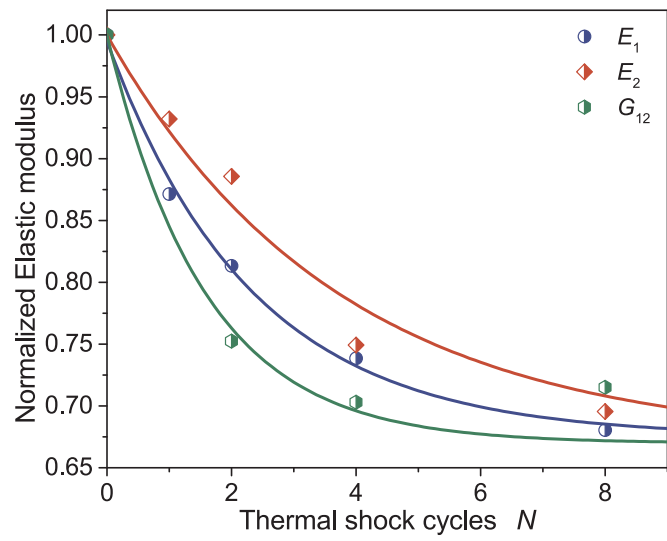

(b) Current elastic moduli as a function of the thermal shock cycle number $N$ with a constant shock temperature $T_{\text {shock }}=$ $1100^{\circ} \mathrm{C}$.

Fig. 4. Correlations between the residual elastic moduli and the thermal shock temperature $T_{\text {shock }}$ and cycle number $N$, respectively. The degradation of the material property influenced by thermal shocks is anisotropic. Modulus values are normalized according to their respective initial values. 


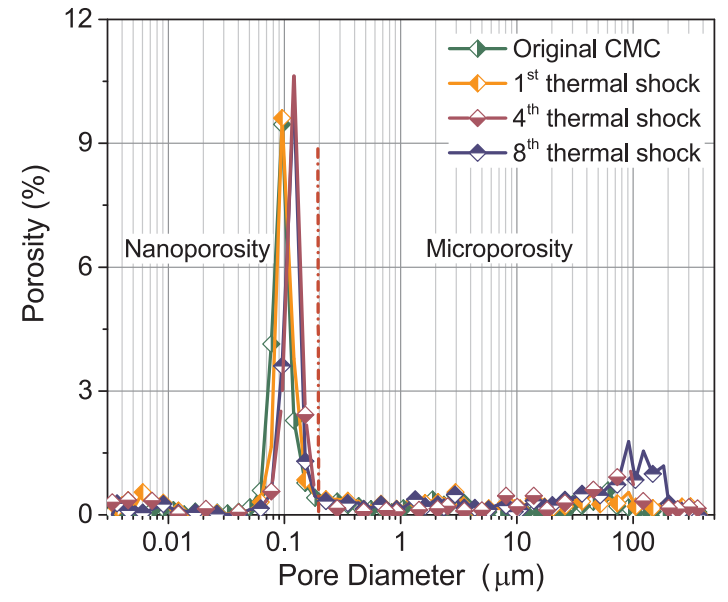

(a) Porosity vs. pore diameter.

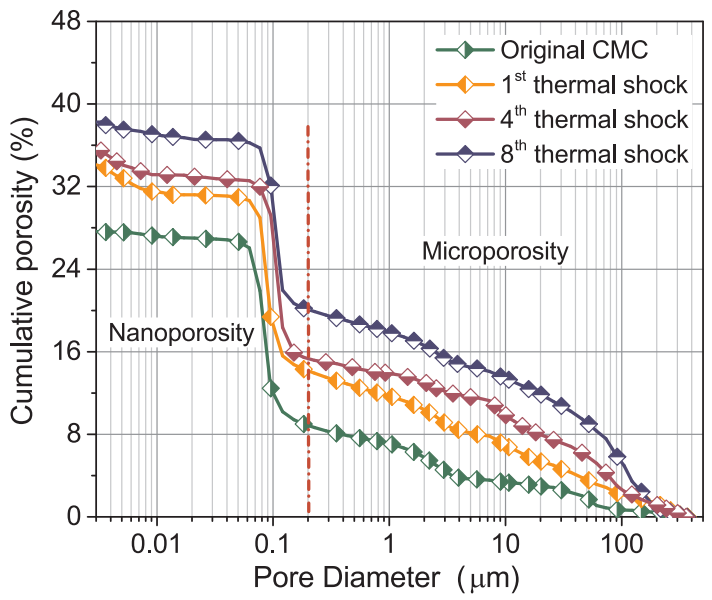

(b) Cumulative porosity vs. pore diameter.

Fig. 5. Hierarchical porosity in thermal shocked specimens determined by the mercury intrusion porosimetry. The thermal shock temperature equals $1100^{\circ} \mathrm{C}$.

changes in the material. Since the fibers are not damaged in the loading process, the damage occurs in the matrix only. The matrix particles are hard and brittle, the damage in the matrix can only in the form of micro-cracks distributed between particles, that is, the increment of the micro porosity represents the micro-cracks due to thermal shocks,

$$
\Delta \zeta=\zeta-\zeta_{0}
$$

where $\zeta$ denotes the current porosity and $\zeta_{0}$ is the initial porosity of the composite. $\Delta \zeta$ stands for porosity increment. Since there are two different populations of pores, $\zeta$ denotes both nano- and micro-pores. Since the micro-cracking is irreversible, $\zeta$ is a monotonic function of the shock cycle number.

In Fig. 6 different porosities of the CMC material are plotted as functions of thermal shock cycles. Whereas the nano-porosity is hardly influenced by thermo-mechanical loads, the micro-pores representing micro-cracks increases with thermal shocks rapidly. The small decreasing of the nano-porosity after a single thermal shock can be understood as transferring the nano-pores into micro-cracks. The increment of the micro-pores can be approximated by a logarithmic function of the thermal shock number,

$$
\Delta \zeta=\alpha \zeta_{0}[\ln (N+1)]^{m_{\mathrm{s}}}
$$

where $\alpha$ is a dimensionless increasing function of the thermal shock temperature, $\ln \left(T_{\text {shock }} / T_{\text {init }}\right)$. Here $\alpha$ equals 0.48 and 0.65 for $T_{\text {shock }}=800^{\circ} \mathrm{C}$ and $T_{\text {shock }}=1100^{\circ} \mathrm{C}$, respectively, which proves that thermal shock temperature is directly related to thermo-mechanical damage in the ceramic matrix composite. $T_{\text {init }}$ is the threshold temperature initiating the thermal shock-induced thermomechanical damage, and equal to ca. $400{ }^{\circ} \mathrm{C}$ for the present oxide/oxide CMC, which implies increasing material damage in the matrix. $m_{\mathrm{s}}$ is the fitting parameter for the approximation. Eq. (4) describes the porosity increment as a function of an increasing cumulative number of thermal shock cycles. Especially the first shock brings dramatically more pores into the material (ca. $30 \%$ of the initial pores), with increasing thermal shocks, the porosity reaches a saturation phase. The total porosity increment is the sum of

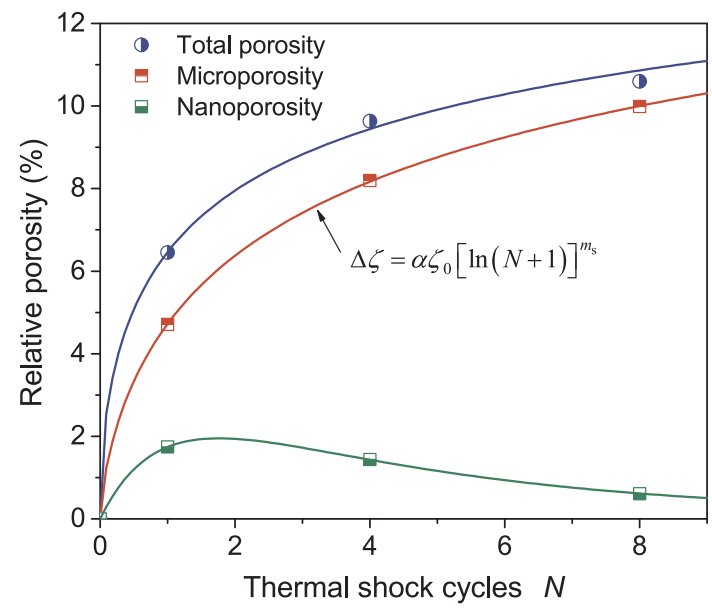

Fig. 6. Porosities as a functions of the thermal shock cycle number $N$ for the shock temperatures equal to $1100^{\circ} \mathrm{C}$. 
the nano-porosity and micro porosity.

\subsection{Effects of porosity}

The porous matrix possesses different mechanical behavior from alumina, which is furthermore affected by the porosity. With increasing porosity, the effective loading area of the material decreases, which implies both material stiffness and material strength reduce with porosity. Mechanical property, such as elastic modulus $E$, yield stress $\sigma_{0}$, ultimate stress $\sigma_{\mathrm{u}}$ and fracture strain $\varepsilon_{\mathrm{f}}$, can empirically be expressed as a power-law function of the mass density [30-34], as

$$
\frac{P}{P_{0}}=\left(\frac{\rho}{\rho_{0}}\right)^{m}=(1-\zeta)^{m}
$$

with the mechanical property $P$ as a function of the mass density $\rho . P_{0}$ stands for the mechanical property of the pore-free mass density $\rho_{0}$. Here the mass density is related to the porosity through $\rho=\rho_{0}(1-\zeta)$ with $\zeta$ as porosity of the material. The exponent $m>1$ depends on the material and varies with mechanical properties [30]. The mechanical property of the fiber phase can be estimated in the same way.

The macroscopic material property of CMC can be estimated based on the rule of mixtures. A general rule of mixtures which is popular in material science is a weighted mean used to predict various properties of a composite material made up of continuous and unidirectional fibers [22]. It provides a theoretical upper- and lower-bound of the properties, such as elastic modulus, mass density, ultimate tensile strength, etc. The axial loading as the upper bound (Voigt model) and the transverse loading as lower bound (Reuss model),

$$
\begin{aligned}
& E_{\mathrm{Up}}=\bar{E}_{\mathrm{m}} V_{\mathrm{m}}+\bar{E}_{\mathrm{f}} V_{\mathrm{f}}, \\
& E_{\mathrm{Low}}=\frac{\bar{E}_{\mathrm{f}} \bar{E}_{\mathrm{m}}}{\bar{E}_{\mathrm{f}} V_{\mathrm{m}}+\bar{E}_{\mathrm{m}} V_{\mathrm{f}}},
\end{aligned}
$$

where $E_{\mathrm{Up}}$ and $E_{\mathrm{Low}}$ are the theoretical upper and lower bound of the composite elastic modulus obtained from the rule of mixture, respectively. $\bar{E}_{\mathrm{f}}=E_{\mathrm{f}}\left(1-\zeta_{\mathrm{f}}\right)^{n_{\mathrm{f}}}$ is the nominal elastic modulus of the fiber phase depending on porosity in the fiber ply, $\zeta_{\mathrm{f}}$. $\bar{E}_{\mathrm{m}}=E_{\mathrm{m}}\left(1-\zeta_{\mathrm{m}}\right)^{n_{\mathrm{m}}}$ represents the nominal elastic modulus of the matrix, which is determined by the particles of alumina and 3YSZ, as shown in Table 1, and by the matrix porosity, $\zeta_{\mathrm{m}}$. $V_{\mathrm{f}}$ and $V_{\mathrm{m}}$ the volume fractions of fiber and matrix in the composite, respectively, and $V_{\mathrm{f}}=1-V_{\mathrm{m}}$.

Based on the power-law for porosity, the rule of mixture can be re-written into

$$
E=\eta_{\mathrm{o}}\left(1-\zeta_{\mathrm{f}}\right)^{n_{\mathrm{f}}} E_{\mathrm{f}} V_{\mathrm{f}}+\left(1-\zeta_{\mathrm{m}}\right)^{n_{\mathrm{m}}} E_{\mathrm{m}} V_{\mathrm{m}}
$$

with $\zeta_{\mathrm{f}}+\zeta_{\mathrm{m}}=\zeta$. Above $\eta_{\mathrm{o}}$ is introduced to describe the effects of the fiber orientation, as suggested in [31]. $n_{\mathrm{f}}$ and $n_{\mathrm{m}}$ are the model parameters for the fiber and matrix, with $n_{\mathrm{f}}=3.15$ and $n_{\mathrm{m}}=24.81$ for the present material. The model is a direct combination of the power-law with the rule of mixture, in analogy to [31], and can be used to predict the property of the porous composite. Fig. 7 shows the composites stiffness degeneration as a function of porosity subject to cyclic thermal shocks. The results confirm a good agreement between the model and the experiments. Both elastic moduli in $0^{\circ}$ and $90^{\circ}$ agree with Eq. (8) well based on the unique model parameters $n_{\mathrm{f}}$ and $n_{\mathrm{m}}$ as well as the fiber orientation factor $\eta_{0}$. The shear modulus was calculated from Eq. (2), that is,

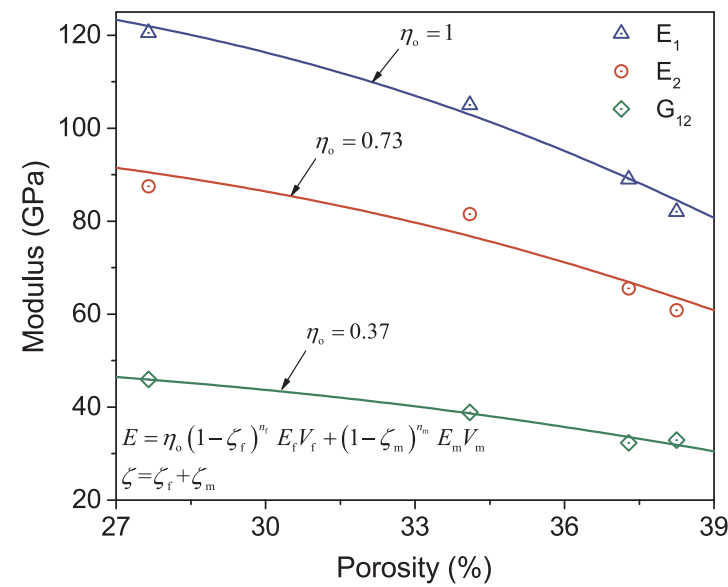

(a)

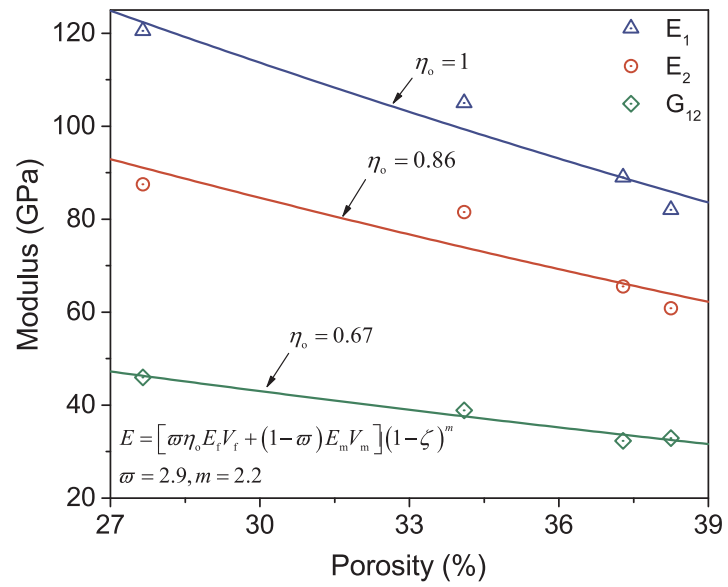

(b)

Fig. 7. Comparison between experimental measurements and predictions from the present models for the correlation between elastic modulus and porosity in different loading directions. (a) Predictions from Eq. (8). (b) Predictions from Eq. (10). 


$$
G_{12}=\frac{E_{x}}{2\left(1-\nu_{21}\right)},
$$

where $E_{x}$ denotes elastic modulus taken from the off-axis tensile test and $\nu_{21}=\varepsilon_{y} / \varepsilon_{x} . x$ is the tension direction. The results confirm that the present CMC mixture rule can describe the macroscopic elastic modulus well.

In Eq. (8) elastic modulus is affected by porosities of both fiber ply and matrix, respectively. The model seems to reflect the variations correctly but is inconvenient for application. By considering the total porosity, the modified rule of mixture can be written as

$$
E=\left[\varpi \eta_{\mathrm{o}} E_{\mathrm{f}} V_{\mathrm{f}}+(1-\varpi) E_{\mathrm{m}} V_{\mathrm{m}}\right](1-\zeta)^{m},
$$

where $\varpi$ is a weight factor for representing the influence of different phases. The fitting parameter $m=2.2$ was found for all tested porosities uniquely. The agreement between the experiments and Eq. (10) is acceptable, as shown in Fig. 7(b). In comparing with Fig. 7(a) the difference between the two models is minor.

\subsection{Representation of material degradation}

In phenomenological damage mechanics, the material degradation is represented by the reduction of the material stiffness, i.e. $D=1-E / E_{0}$ with $E$ as the current elastic modulus and $E_{0}$ as the initial modulus. Such a formulation is easy to implement to describe damage processes [35,36]. However, the damage variable becomes a tensor of second order for composites [16,17]. The damage equation becomes rather complex. Schmücker [37] confirmed that the microstructure and mechanical property of Nextel ${ }^{\mathrm{TM}}$ 610/ alumina fibers are stable up to $1200^{\circ} \mathrm{C}$, even in water vapor environment. That is, the present composite failure is dominated by the matrix damage, whereas the fibers remain undamaged. The oxide/oxide CMC is a material with a weak matrix, which can be learned from the significant difference of the on-axis tension stiffness from the off-axis stiffness [15]. Such materials are damaged simply due to nucleation and propagation of micro-cracks in the matrix [13]. $D$ for the present CMC material represents the reduction of the average stiffness and is not directly related to the micro-cracks in the matrix.

Assuming that the elastic modulus of matrix is reduced $10 \%$, the variation of the average material stiffness can be estimated from Eq. (8) and follows $D=0.058 \%$, i.e. ca. twenty-times difference from the matrix degradation. This result implies that the description based on the average elastic modulus cannot represent the CMC damage accurately, due to very low sensitivity. A variable directly related to the material micro-structure, such as the micro-porosity in the matrix, may give a more reliable description of the damage behavior.

\section{The damage evolution model depending on porosity}

In the process of thermal shocks, rapid temperature changes result in severe local thermal stresses and lead to cracks in the matrix as well as delaminations in fiber plies. From fracture mechanics cracks are driven by the strain energy. The energy release rate describes crack propagation. Micro-cracks in composites can be described by the energy release rate [23,38-40]. The material degradation is a collective phenomenon of micro-crack propagations. As discussed previously, micro-cracks induced by the thermomechanical loading are the damage mechanism of the CMC failure and can be related to the material's porosity. Establishing quantitative correlation between the porosity and the applied thermo-mechanical load is of importance for understanding mechanical behavior and application of the oxide/oxide CMC.

According to the idea of Kachanov the material damage can be represented by the load bearing area in the material [35]. The crack driving force, such as energy release rate, is defined as

$$
G=\frac{\partial U}{\partial A_{\text {crack }}},
$$

where $A_{\text {crack }}$ denotes the total crack surface in the loading direction. $U$ represents the strain energy of the specimen, as [39,41]

$$
U=\frac{1}{2} \frac{\sigma_{\text {th }}^{2}}{E} V
$$

for the thermal shocked specimen. Above $E$ is the current elastic modulus of the damaged material, $\sigma_{\text {th }}$ is the thermal stress under thermal shocks, and $V$ is the representative volume of interest. If $V$ is small enough, $A_{\text {crack }}$ can be represented by

$$
A_{\text {crack }}=\frac{V_{\text {crack }}}{s}=\frac{\zeta V}{s}
$$

with $s$ as the nominal width of micro-cracks in the representative volume $V . V_{\text {crack }}=\zeta V$ is the pore volume in the specimen. For an arbitrarily small volume $V$, the representative crack width $s$ can be assumed as constant, for instance, with $s=1.5 \mu$ m, as suggested by $[42,43]$.

In thermal shocks, the thermal stress $\sigma_{\text {th }}$ in the specimen causes damage. According to [13], $\sigma_{\text {th }}$ is proportional to the temperature $T_{\text {shock}}$. The thin plate possesses a biaxial stress state with the in-plane stresses equal to $\sigma_{\text {th }}$ and the out-of-plane stress approximately vanishes. Under the assumption of the elastic damage for the oxide/oxide CMC material, the thermal stress in the specimen can be written as [13] 


$$
\sigma_{\mathrm{th}}=\frac{\alpha_{\mathrm{TEC}} T_{\mathrm{shock}} E}{1-\nu} \psi\left(B_{\mathrm{i}}\right)
$$

with a correction term $\psi\left(B_{\mathrm{i}}\right)$ depending on both convection and plate geometry. $\alpha_{\mathrm{TEC}}$ is the thermal expansion coefficient (TEC). For relatively low values of $B_{\mathrm{i}}$, the following semi-empirical equation was suggested for the surface stress calculation [44],

$$
\psi\left(B_{\mathrm{i}}\right)=\frac{B_{\mathrm{i}}}{3.25}, \quad B_{\mathrm{i}}=\frac{h l}{k}
$$

for $\psi\left(B_{\mathrm{i}}\right)$ in range from 0 to 1 . Above $B_{\mathrm{i}}$ is the Biot modulus, $h$ is the heat transfer coefficient, $k$ is the thermal conductivity, and $l$ is the half thickness of the thin plate specimen. From the cyclic thermal shock experiments, the elastic modulus variation can be directly measured and the thermal stress $\sigma_{\text {th }}$ can be calculated from Eq. (14).

The energy release rate for the thermo-mechanical damage process can be derived from the equations above, as

$$
G=\frac{\partial U}{\partial A_{\text {crack }}}=s \frac{\partial}{\partial \zeta}\left(\frac{1}{2} \frac{\sigma_{\mathrm{th}}^{2}}{E}\right)
$$

Substituting the expressions above into Eq. (16), the expression for thermal shocks can be further simplified into

$$
G=m \gamma(1-\zeta)^{m-1}
$$

where the strain energy release rate $G$ is affected by the parameter $\gamma$ defined as

$$
\gamma=\frac{s}{2}\left[\frac{\alpha_{\mathrm{TEC}} T_{\text {shock }}}{1-\nu} \psi\left(B_{\mathrm{i}}\right)\right]^{2}\left[\varpi \eta_{\mathrm{o}} E_{\mathrm{f}} V_{\mathrm{f}}+(1-\varpi) E_{\mathrm{m}} V_{\mathrm{m}}\right]
$$

summarized in Table 2 dependning on the orientation. Above the rule of mixture, Eq. (10) is used. The total porosity $\zeta=\zeta_{0}+\Delta \zeta$.

Fig. 8 illustrates the strain energy release rate $G$ as a function of the porosity increment $\Delta \zeta$ in the 2-D woven oxide/oxide CMC after cyclic thermal shocks. The strain energy release rate $G$ of the damaged material decreases with thermo-mechanical accumulative damage. The experiments agree with model curves excellently in all orientations. It should be noted that the thermo-mechanical damage evolution focus on the mechanical behavior of the oxide/oxide CMC after thermal shocks rather than during the thermal shock process.

\section{Conclusions}

In the present work, the thermo-mechanical damage evolution from cyclic thermal shocks in the 2-D woven oxide/oxide ceramic matrix composite has been investigated experimentally. A novel characterization approach based on the micro porosity variation is developed for cyclic thermal shock-induced thermo-mechanical damage. From the present investigation the following conclusions can be drawn:

- The thermal shock tests confirmed that the thermo-mechanical damage in the present CMC material is anisotropic. Due to cyclic thermal shocks, the mechanical property in different directions shows significantly different variations. The representation of the damage by the material stiffness reduction needs a tensor-valued function.

- The present work introduced the porosity increment as the damage indicator and established unique correlations for material degradation in different loading directions. The model predictions agree with all experiments performed in the present work. The anisotropic damage can be described by the porosity variation alone.

- The strain energy release rate of the damaged CMC is related to the porosity in a power-law function, in which the loading orientation influence is considered in an orientation-dependent parameter. The model provides an excellent agreement to experiments.

- Effects of the hierarchical porosity to elastic moduli are expressed by a modified rule of mixture for different specimen orientations under various damage states. The porosity gives a uniform and much more sensitive description of the material stiffness variation.

\section{Acknowledgement}

The present work is financed by the China Natural Science Foundation under the contract numbers 11572169 and 51775294.

Table 2

Effect of fiber orientation to the strain energy release rate $G$ (Thermal shock temperature $T_{\text {shock }}=1100{ }^{\circ} \mathrm{C}$ )

\begin{tabular}{cccc}
\hline Fiber orientation & $0^{\circ}$ & $90^{\circ}$ & $45^{\circ}$ \\
\hline$\gamma\left(\mathrm{kJ} / \mathrm{m}^{2}\right)$ & 0.20 & 0.15 & 0.08 \\
$G\left(\mathrm{~kJ} / \mathrm{m}^{2}\right)$ & 0.306 & 0.228 & 0.116 \\
\hline
\end{tabular}




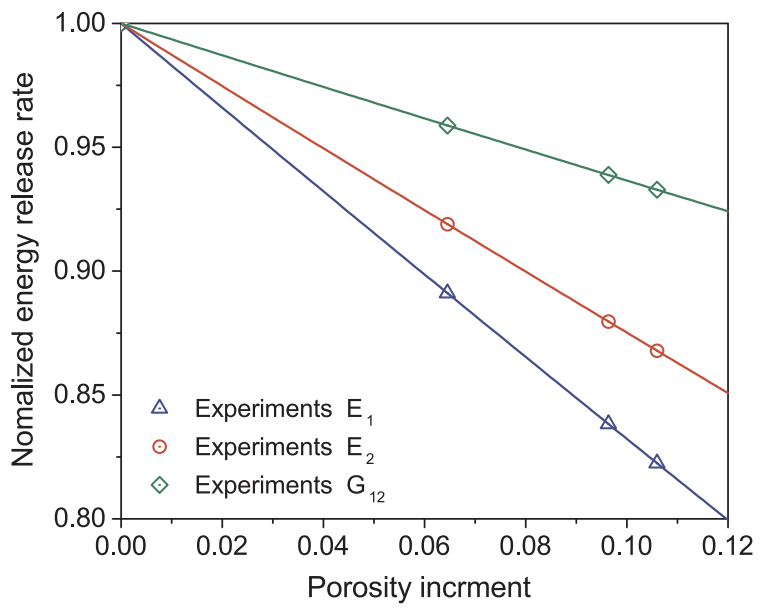

Fig. 8. Correlation between the energy release rate of the material with porosity increment $\Delta \zeta$ in the thermal shocked composites.

\section{Appendix A. Supplementary material}

Supplementary data associated with this article can be found, in the online version, at https://doi.org/10.1016/j.engfracmech. 2019.106669.

\section{References}

[1] Rajan VP, Zok FW. Stress distributions in bluntly-notched ceramic composite laminates. Compos Part A-Appl Sci Manuf 2014;60(Supplement C):15-23.

[2] Paradkar AG, Shanti Ravali N, Eswara Prasad N. Mechanical behavior of 2D and 3D weaved SiC-matrix, carbon-continuous-fibre-reinforced composites: Part 2. fracture toughness under static loading conditions. Eng Fract Mech 2017;182:52-61.

[3] Vasechko V, Flucht F, Rahner N. Mechanical investigation of weak regions in a wound oxide-oxide ceramic matrix composite. J Eur Ceram Soc 2018;38(15):5192-9.

[4] Lamon J, Thommeret B, Percevault C. Probabilistic-statistical approach to matrix damage and stress-strain behavior of 2-D woven SiC/SiC ceramic matrix composites. J Eur Ceram Soc 1998;18(13):1797-808.

[5] Jackson PR, Ruggles-Wrenn MB, Baek SS, Keller KA. Compressive creep behavior of an oxide-oxide ceramic composite with monazite fiber coating at elevated temperatures. Mater Sci Eng: A 2007;454-455:590-601.

[6] Gowayed Y, Pierce J, Buchanan D, Zawada L, John R, Davidson K. Effect of microstructural features and properties of constituents on the thermo-elastic properties of ceramic matrix composites. Compos Part B-Eng 2018;135:155-65.

[7] Moser B, Rossoll A, Weber L, Beffort O, Mortensen A. Damage evolution of Nextel ${ }^{\mathrm{TM}} 610$ alumina fibre reinforced aluminium. Acta Mater 2004;52(3):573-81.

[8] Mall S, LaRochelle KJ. Fatigue and stress-rupture behaviors of SiC/SiC composite under humid environment at elevated temperature. Compos Sci Technol 2006;66(15):2925-34.

[9] Li L. Synergistic effects of temperature, oxidation, loading frequency and stress-rupture on damage evolution of cross-ply ceramic-matrix composites under cyclic fatigue loading at elevated temperatures in oxidizing atmosphere. Eng Fract Mech 2017;175:15-30.

[10] Sun J, Yuan H. Life assessment of multiaxial thermomechanical fatigue of a nickel-based superalloy inconel 718. Int J Fatigue 2019;120(3):226-40.

[11] Wang HY, Singh RN, Lowden RA. Thermal shock behavior of two-dimensional woven fiber-reinforced ceramic composites. J Am Ceram Soc 1996;79(7):1783-92.

[12] Boccaccini AR, Strutt AJ, Vecchio KS, Mendoza D, Chawla KK, Ponton CB, et al. Behavior of nicalon ${ }^{\text {TM }}$-fiber-reinforced glass-matrix composites under thermal cycling conditions. Compos Part A: Appl Sci Manuf 1998;29(11):1343-52.

[13] Yang Z, Yuan H, Liu H. Evolution and characterization of cyclic thermal shock-induced thermomechanical damage in oxide/oxide ceramics matrix composites. Int J Fatigue 2019;120:150-61.

[14] Yang Z, Yuan H, Markert B. Representation of micro-structural evolution and thermo-mechanical damage in thermal shocked 2-D woven oxide/oxide ceramic matrix composites. Int J Fatigue 2019;126:122-9.

[15] Liu H, Yang Z, Yuan H. A novel elastoplastic constitutive model for woven oxide/oxide ceramic matrix composites with anisotropic hardening. Compos Struct. in press.

[16] Chaboche JL, Maire JF. A new micromechanics based CDM model and its application to CMC's. Aerosp Sci Technol 2002;6(2):131-45.

[17] Siron O, Pailhes J, Lamon J. Modelling of the stress/strain behaviour of a carbon/carbon composite with a 2.5 dimensional fibre architecture under tensile and shear loads at room temperature. Compos Sci Technol 1999;59(1):1-12.

[18] Talreja R. Stiffness properties of composite laminates with matrix cracking and interior delamination. Eng Fract Mech 1986;25(5):751-62.

[19] Jin ZH, Batra RC. Thermal shock cracking in a metal-particle-reinforced ceramic matrix composite. Eng Fract Mech 1999;62(4):339-50.

[20] Santhosh U, Ahmad J, Ojard G, Miller R, Gowayed Y. Deformation and damage modeling of ceramic matrix composites under multiaxial stresses. Compos Part BEng 2016;90:97-106.

[21] Zawada LP, Hay RS, Lee SS, Staehler J. Characterization and high-temperature mechanical behavior of an oxide/oxide composite. J Am Ceram Soc 2003;86(6):981-90.

[22] Askeland DR, Fulay PP, Wright WJ. The science and engineering of materials. 6th ed. Cengage Learning; 2010.

[23] Yuan H, Li X. Critical remarks to cohesive zone modeling for three-dimensional elastoplastic fatigue crack propagation. Eng Fract Mech 2018;202:311-31.

[24] Pei C, Shi D, Yuan H, Li H. Assessment of mechanical properties and fatigue performance of a selective laser melted nickel-base superalloy inconel 718. Mater Sci Eng: A 2019;759(3):278-87.

[25] Volkmann E, Tushtev K, Koch D, Wilhelmi C, Goring J, Rezwan K. Assessment of three oxide/oxide ceramic matrix composites: mechanical performance and effects of heat treatments. Composites Part: A 2015;68(Supplement C):19-28.

[26] ASTM-D3518, Standard test method for in-plane shear response of polymer matrix composite materials by tensile test of a \pm 45laminate, American Society for Testing and Materials D3518/D3518M; 2001.

[27] Gélébart L, Chateau C, Bornert M, Crépin J, Boller E. X-ray tomographic characterization of the macroscopic porosity of chemical vapor infiltration SiC/SiC composites: effects on the elastic behavior. Int J Appl Ceram Technol 2010;7(3):348-60. 
[28] Wilson DM, Visser LR. High performance oxide fibers for metal and ceramic composites. Compos Part A 2001;32(8):1143-53.

[29] Levi CG, Yang JY, Dalgleish BJ, Zok FW, Evans AG. Processing and performance of an all-oxide ceramic composite. J Am Ceram Soc 2005;81(8):2077-86.

[30] Yuan H, Zhang L, Ma S. Damage evolution and characterization for sintered powder metals with the varying porosity. Eng Fract Mech 2019;207:86-98.

[31] Madsen B, Thygesen A, Lilholt H. Plant fibre composites-porosity and stiffness. Compos Sci Technol 2009;9:1057-69.

[32] Aguilar C, Arancibia M, Alfonso I, Sancy M, Tello K, Salinas V, et al. Influence of porosity on the elastic modulus of Ti-Zr-Ta-Nb foams with a low nb content. Metals 2019;9:15.

[33] Ma S, Yuan H. Computational investigation of multi-axial damage modeling for porous sintered metals with experimental verification. Eng Fract Mech 2015;149(4):89-110.

[34] Li H, Li J, Yuan H. A review of the extended finite element method on macrocrack and microcrack growth simulations. Theoret Appl Fract Mech 2018;97(4):236-49.

[35] Lemaitre J, Desmorat R. Engineering damage mechanics: ductile, creep, fatigue and brittle failures. Berlin Heidelberg: Springer-Verlag; 2005.

[36] Ma S, Yuan H. A continuum damage model for multi-axial low cycle fatigue of porous sintered metals based on the critical plane concept. Mech Mater 2017;104:13-25.

[37] Schmücker M, Flucht F, Mechnich P. Degradation of oxide fibers by thermal overload and environmental effects. Mater Sci Eng: A 2012;557:10-6.

[38] Gross D, Seelig T. Fracture mechanics-with an introduction to micromechanics, continuum mechanics and mechanics of materials. Springer International Publishing; 2018.

[39] Siulie L, Nairn JA. The formation and propagation of matrix microcracks in cross-ply laminates during static loading. J Reinf Plast Compos 1992;11(2):158-78.

[40] Zhang Y, Guo L, Huang K, Bai X, Pang J, Zhang Z. A numerical method for the thermal-shock crack problems of nonhomogeneous materials with inclusions based on an interaction energy integral method. Eng Fract Mech 2018;190:159-74.

[41] Vinogradov V, Hashin Z. Probabilistic energy based model for prediction of transverse cracking in cross-ply laminates. Int J Solids Struct 2005;42(2):365-92.

[42] Dharani LR, Chai L, Pagano NJ. Steady-state cracking in ceramic matrix composites. Compos Sci Technol 1990;39(1):29-43.

[43] Vinet C, Priou P. Micromechanical damage model taking loading-induced anisotropy into account. Aerosp Sci Technol 1997;1(1):65-76.

[44] Kingery WD. Factors affecting thermal stress resistance of ceramic materials. J Am Ceram Soc 1955;38(1):3-15. 